\title{
Bufalin enhances anti-angiogenic effect of sorafenib via AKT/VEGF signaling
}

\author{
HAIYONG WANG ${ }^{1,2^{*}}$, CHENYUE ZHANG ${ }^{1,2^{*}}$, ZHOUYU NING $^{1,2}$, \\ LITAO XU ${ }^{1,2}$, XIAOYAN ZHU ${ }^{1,2}$ and ZHIQIANG MENG ${ }^{1,2}$ \\ ${ }^{1}$ Department of Integrative Oncology, Fudan University Shanghai Cancer Center; \\ ${ }^{2}$ Department of Oncology, Shanghai Medical College, Fudan University, Shanghai 200032, P.R. China
}

Received October 17, 2015; Accepted November 22, 2015

DOI: 10.3892/ijo.2016.3326

\begin{abstract}
Sorafenib mainly exerts its anti-hepatoma effect by inhibiting tumor angiogenesis. However, its curative effect is limited. Thus, application of drugs which could augment its anti-angiogenic effect is necessary. Bufalin has been reported to possess anticancer properties. In the present study, we investigated the synergistic anti-angiogenic effect of sorafenib combined with bufalin. The enhanced anti-angiogenic effect of the combination treatment was firstly assessed in nude mice bearing human HCC intradermal tumors. In addition, we found that proliferation was significantly inhibited and the morphology was obviously changed in the combinationtreated human umbilical vein endothelial cells (HUVEC) at $48 \mathrm{~h}$ of treatment. In addition, the combination treatment was found to suppress vessel formation potently as proved in the tube formation, chick chorioallantoic membrane and rat aortic rings. Mechanistically, HUVEC incubated with the combination treatment showed increased apoptosis, decreased migration, which might account for its capacity against angiogenesis. Vascular endothelial cells have been reported to secrete cytokines to affect angiogenesis. Therefore, suspensions from HUVECs with different treatments were collected as conditioned medium (CM). The combinationtreated CM significantly inhibited the migration of HUVEC and blood vessel formation in vitro. Importantly, multiple cytokines associated with angiogenesis were downregulated in the combination-treated CM. Furthermore, we verified that the secretion of VEGF was downregulated and revealed that the reduction might be regulated through the inhibition of the PI3K/AKT pathway. Taken together, our findings demonstrated for the first time that bufalin can enhance
\end{abstract}

Correspondence to: Professor Zhiqiang Meng, Department of Integrative Oncology, Fudan University Shanghai Cancer Center, 270 Dong An Road, Shanghai 200032, P.R. China

E-mail: mengzhq@gmail.com

${ }^{*}$ Contributed equally

Key words: bufalin, sorafenib, angiogenesis, AKT/VEGF anti-angiogenic effect of sorafenib via modulating the AKT/ VEGF signaling pathway.

\section{Introduction}

HCC has been recognized as a hypervascular cancer (1). The growth of a liver tumor requires the formation of new blood vessels, which has provided a strong rationale for antiangiogenic strategies as therapy $(2,3)$. Angiogenesis plays an important role in tumor growth by supplying nutrients and providing a route for tumor growth, invasion and metastasis $(4,5)$. Therefore, targeting tumor angiogenesis might be a good option for HCC treatment. Moreover, a series of signaling pathways participating in the development of microvasculacture have been identified (5).

Studies have shown that VEGF is strongly involved in the development of liver tumor vascularization and the infiltration of cancer cells into the tumor capsule in HCC $(2,6)$. VEGF can be derived from different sources such as stromal cells, extracellular matrix, and cancer cells. The VEGF/VEGFR signaling pathway is essential for drawing endothelial cells from pre-existing blood vessels and in stimulating their growth. A number of studies have shown that the vascular endothelial cells can affect angiogenesis by induction of a number of cytokines such as VEGF $(7,8)$. Therefore, suppression of VEGF released from endothelial cells is of vital importance in the inhibition of tumor angiogenesis, which may achieve enhanced tumor shrinkage.

Previous studies have shown that PI3K/AKT signaling regulates angiogenesis through affecting the expression of VEGF (9-12). It may contribute to tumor angiogenesis via a paracrine pathway to the surrounding microvessels by targeting endothelial cells $(13,14)$. In endothelial cells, the majority of growth factor-induced responses are mediated by the activation of the PI3K-AKT signaling cascade $(15,16)$. First, AKT is known to mediate hypoxia-induced expression of VEGF in vitro and in vivo $(17,18)$. Studies suggest that the level of active AKT, as well as its short-term and long-term activation states, in vascular cells can regulate various signaling pathways to affect the balance of pro- and anti-angiogenic factors (19-21). In addition to VEGF, AKT might affect the protein levels and activities of several key regulators of angiogenesis, including Anhiopoetin $(15,22)$. Thus, AKT/VEGF 
pathway may constitute a therapeutic target for angiogenesis anomaly in HCC.

Sorafenib, serving as a tyrosine kinase inhibitor, mainly exerts its anti-angiogenic effect by targeting VEGFR-2, -3, PDGFR. However, its effect on inhibiting angiogenesis is limited, as it only improves overall survival of HCC patients by no more than three months $(23,24)$. Thus, combination with other drugs which may augment the anti-angiogenic effect of sorafenib should be encouraged.

Bufalin, extracted from Chinese herbs, has been proven to induce apoptosis, inhibit proliferation and metastasis among various cancers (25). However, little information exists concerning the anti-angiogenic role of bufalin. An earlier study has demonstrated that the in vitro angioinhibitory action of bufalin may be induced by the proliferation inhibition of endothelial cells through the arrest at the G2/M phase of a cell cycle (26).

Still, it was unknown whether bufalin could enhance anti-angiogenic effect of sorafenib. Moreover, the underlying mechanisms involved in this process remain to be clearly defined. Therefore, in the present study we investigated bufalin-mediated regulation of angiogenesis and whether it could enhance the anti-angiogenic effect of sorafenib.

\section{Materials and methods}

Reagents and antibodies. Bufalin and dimethyl sulfoxide (DMSO) were obtained from Sigma Chemical Co. (St. Louis, MO, USA). Sorafenib was purchased from Bayer Corp. (West Haven, CT, USA). Antibodies against VEGF were purchased from Bioworld Technology (Minneapolis, MN, USA). The specific primary antibodies for mTOR, phosphorylated-mTOR (p-mTOR), AKT, phosphorylated-AKT (p-AKT), ERK1/2, phosphorylated-ERK1/2 (p-ERK1/2), CD31, and GAPDH were purchased from Cell Signaling Technology (Danvers, MA, USA); The PI3K/AKT inhibitor PI103 was purchased from Selleck Chemicals LLC (Houston, TX, USA). Human Angiogenesis Array Q1 was purchased from RayBiotech (Norcross, GA, USA).

Cell culture. HUVECs originated from the American Type Culture Collection (ATCC) and were cultured in DMEM containing $10 \%$ fetal bovine serum (FBS; Biochrom, Berlin, Germany) in $5 \% \mathrm{CO}_{2}$ at $37^{\circ} \mathrm{C}$. The human $\mathrm{HCC}$ cell lines SMMC7721 also originated from the American Type Culture Collection (ATCC) and were cultured in RPMI-1640 containing $10 \% \mathrm{FBS}$ in $5 \% \mathrm{CO}_{2}$ at $37^{\circ} \mathrm{C}$.

Abdominal tumor model. Six-week-old BALBc nu/nu mice were obtained from the Shanghai Institute of Material Medica, Chinese Academy of Science. The mice were bred in laminar flow cabinets under pathogen-free conditions. We followed internationally recognized guidelines on animal welfare. The study design was approved by the Animal Ethics Committee, and the experiments were undertaken in accordance with the ethical principles of the Animal Experimentation of Fudan University. The SMMC7721 cells $\left(5 \times 10^{6}\right)$ were subcutaneously inoculated into the abdominal intraderma of 6-week-old $\mathrm{BALBc}$ nu/nu mice. According to tumor size, the mice were randomly separated into four groups with three mice per group. The mice in the experimental group received intraperitoneal injections of $1 \mathrm{mg} / \mathrm{kg}$ bufalin (5 days/week), oral uptake of $30 \mathrm{mg} / \mathrm{kg} /$ day sorafenib (5 days/week), and the combination of both intraperitoneal injections of bufalin and oral uptake of sorafenib. The control mice were injected with the vehicle only. The treatment was continued for two weeks. Then, the mice were sacrificed, and the tumors and blood vessel were observed.

Subcutaneous tumor model. To determine the in vivo antiangiogenic activity of the combination treatment, viable SMMC7721 cells $\left(5 \times 10^{6}\right)$ were subcutaneously inoculated into the right flank of 6 -week-old BALBc nu/nu mice. When the average subcutaneous tumor volume reached $100-300 \mathrm{~mm}^{3}$, the mice were randomly divided into four groups as indicated, with six mice per group. Tumor size was measured every four days after the treatment. Tumor-bearing mice were sacrificed after 16 days of treatment, and the tumor weight was evaluated.

Cell viability assay. The cell proliferation analysis was performed as previously described (27). Briefly, cells were plated at 5000 cells per well in 96-well microtiter plates and incubated overnight at $37^{\circ} \mathrm{C}$ in a humidified incubator containing $5 \% \mathrm{CO}_{2}$. The following day, various concentrations of drugs were added to the wells, and the cultures were incubated for an additional 24,48 , or $72 \mathrm{~h}$. Cell viability was determined using a Cell Counting Kit-8 (Dojindo, Gaithersburg, MD, USA) according to the manufacturer's instructions.

Cell cycle and apoptosis assay. HUVECs were seeded in 6 -well plates at a density of $2 \times 10^{5}$ cells/well and were subjected to different treatments for $48 \mathrm{~h}$. Then cells were trypsinized, washed with PBS and fixed in 70\% methanol. Fixed cells were then washed with PBS, incubated with $100 \mu \mathrm{g} / \mathrm{ml}$ RNAase for $30 \mathrm{~min}$ at $37^{\circ} \mathrm{C}$, stained with propidium iodide $(50 \mu \mathrm{g} / \mathrm{ml})$. Cells were then subjected to flow cytometry (FCM) using Beckman FACScanto. The percentages of cells in different cell cycle phases were analyzed using ModFit LT software. Cell apoptosis was determined by Annexin V and PI staining via FCM. The apoptosis was also observed by PI staining using Hoechst 33258.

Scratch wound assay. HUVECs were seeded in 6-well plates and grown to near $100 \%$ confluency. The cells were scratched with a pipette tip to create wounds. Treatment with bufalin $(2.5 \mathrm{nM})$ and sorafenib $(2.5 \mu \mathrm{M})$ and their combination was given in serum-free medium after the scratch was made. Randomly chosen fields were photographed at a magnification of $\times 10$ with an inverted microscope, and the images were taken at identical locations at $48 \mathrm{~h}$ after treatment. Percentage of wound was calculated by comparing the final gap width to the initial gap width using Image pro-plus.

Cell migration assay. For the cell migration assay, the cell migration was assessed using the Transwell assay (Boyden Chambers, Corning, Cambridge, MA, USA). Cells $\left(5 \times 10^{4}\right)$ were seeded in serum-free medium in the upper chamber and 
allowed to migrate towards the lower chamber that contained $10 \%$ FBS. After $48 \mathrm{~h}$, the cells that had traveled through and adhered to the underside of the membrane were counted at a magnification of $\mathrm{x} 200$.

Tube formation. HUVECs were cultured and subjected to different treatments as described above. At $48 \mathrm{~h}$ after treatment, cell suspensions were collected as conditioned medium (CM). The CM was collected after high speed centrifugation and stored at $-80^{\circ} \mathrm{C}$. After thawed at $4{ }^{\circ} \mathrm{C}$ overnight, the Matrigel was coated in 96-well plate then incubated at room temperature for at least $30 \mathrm{~min}$ to gel. HUVECs were seeded at density of $4 \times 10^{4}$ cells/well/100 $\mu \mathrm{l}$ on the Matrigel. HUVECs were cultured and exposed to different treatments as indicated. After incubation for indicated time, the formed networks at 96-well plate were photographed. Minimum of three fields were analyzed per image.

Chorioallantoic membrane (CAM) of chick embryos assay. The chicken chorioallantoic membrane (CAM) assay was performed using eight-day-old fertilized chicken eggs. A 1-cm diameter window was created in the shell of each egg and the surface of the dermic sheet was removed to expose the CAM. A 0.5-cm diameter filter paper was placed on top of the CAM, and a volume of $100 \mu \mathrm{l}$ drug (control, bufalin, sorafenib, the combination) was placed on the center of the filter paper. Then the windows in the shell were closed using sterilized bandages. The eggs were incubated at $37^{\circ} \mathrm{C}$ at $90 \%$ relative humidity for $48 \mathrm{~h}$. Following fixation with stationary solution (a mixture of methanol and acetone with a volume ratio of 1:1) for $15 \mathrm{~min}$, the CAM was excised and imaged using a digital camera. The morphology of chicken blood vessels with different treatments was detected.

Rat aortic ring assay. Thoracic aortae were first dissected from 8-week-old Wistar rats and were then immediately transferred to a culture dish containing cold DMEM containing $10 \%$ fetal bovine serum. The periaortic fibroadipose tissue was carefully removed with fine microdissecting forceps and iridectomy scissors, paying special attention not to damage the aortic wall. Next, rings were embedded in Matrigel. Once embedded, the rings were fed with DMEM every 2-3 days. Alternatively, different drugs described above were added to the medium for $48 \mathrm{~h}$ to test their effects on angiogenesis. Rings were photographed under clear field illumination by using an inverted microscope.

Detection of cytokines related to angiogenesis using Human Angiogenesis Array. To detect the cytokines related to angiogenesis in the medium of HUVEC treated with different drugs, we performed Human Angiogenesis Array. Cytokines closely linked with angiogenesis were detected. The conditioned media from HUVECs were placed into wells overlaid with antibody specific for these cytokines. After binding with specific antibody, the absorbance was measured at $450 \mathrm{~nm}$ using a microplate reader. The concentrations of different cytokines were evaluated. Specific experimental procedures were executed according to Human Angiogenesis Array Q1 kit instructions.
Enzyme-linked immunosorbent (ELISA) assay. Suspensions from HUVECs with different treatments were collected and the VEGF levels were measured using a sandwich ELISA kit (R\&D Systems, Minneapolis, MN, USA) according to the manufacturer's instructions and analyzed using a Labsystems Multiscan reader (Thermo Fisher Scientific, Waltham, MA, USA).

Western blotting. The HUVECs were exposed to the indicated stimulations and washed with cold PBS and lysed in the culture dishes using a PhosphoSafe ${ }^{\mathrm{TM}}$ Extraction Reagent (Merck, Darmstadt, Germany) containing 1\% Protease Inhibitor Cocktail (EDTA-free, Thermo, San Jose, CA, USA). The protein concentrations were then determined using the Bio-Rad detergent compatible protein assays (Bio-Rad, Hercules, CA, USA). Protein from the control and treated cell lysates was loaded onto 8-12\% gradient NuPAGE gels (Novex, San Diego, CA, USA), electrophoresed under reducing conditions, and transferred onto polyvinylidene difluoride membranes (0.22 $\mu \mathrm{m}$; Millipore). Western blot analysis was performed as previously described (27).

Immunohistochemistry. Tumor samples from in vivo studies were rinsed in PBS and fixed in 10\% paraformaldehyde/PBS. Samples were dehydrated in $70 \%$ ethanol, paraffin embedded, and sectioned $(4 \mu \mathrm{m})$. Then these sections were stained with CD31 antibody. The mean positive staining density was analyzed in the randomly selected areas in each section using image analysis software.

Statistical analysis. The experiments reported herein were repeated in triplicate. Independent Student's t-test was used to analyze the variation of two selected groups. A $P<0.05$ was considered statistically significant and $P<0.01$ was considered highly statistically significant. All statistical analyses were performed with SPSS software.

\section{Results}

Combination treatment inhibits intradermal tumor angiogenesis. In order to observe the effect of the combination treatment on tumor angiogenesis, we used the intradermal tumor angiogenesis model (28). SMMC7721 cell lines implanted intradermally in nude mice were found to induce significant angiogenesis within a period of days until the tumors could be measured. Intraperitoneal injection of bufalin at a dose of $1 \mathrm{mg} / \mathrm{kg} /$ day and oral administration of sorafenib at a dose of $30 \mathrm{mg} / \mathrm{kg} /$ day for consecutive 14 days inhibited blood vessel formation in the intradermal tumors in nude mice, as manifested by the vessel numbers and branches. Not surprisingly, the tumor vessel formation was more inhibited in nude mice with the combination treatment, as reflected in both vessel numbers and branches (Fig. 1A and B). We also found that the tumor weight was significantly attenuated in nude mice with the combination treatment, as compared with the ones treated with either bufalin or sorafenib (Fig. 1C).

Synergistic inhibitory efficacy of combination treatment on proliferation of HUVEC in vitro. HUVECs were subjected to the indicated treatments. They were incubated with either 
A

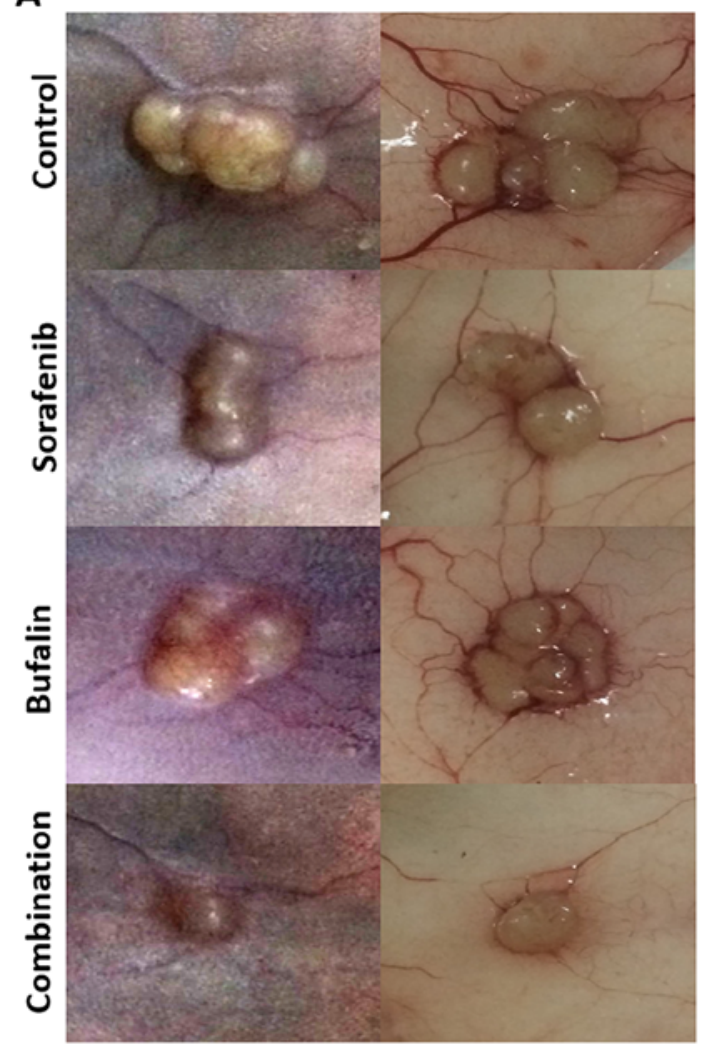

B

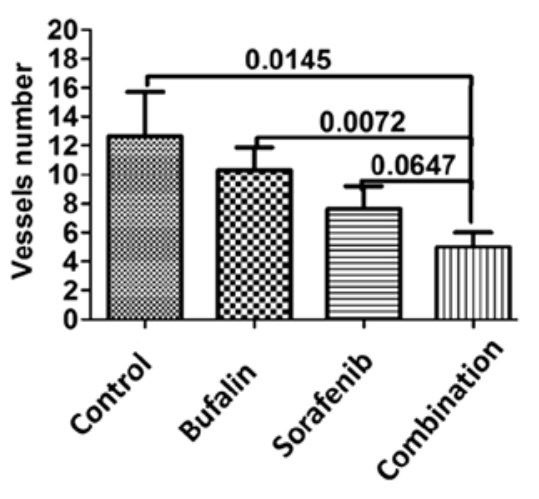

C

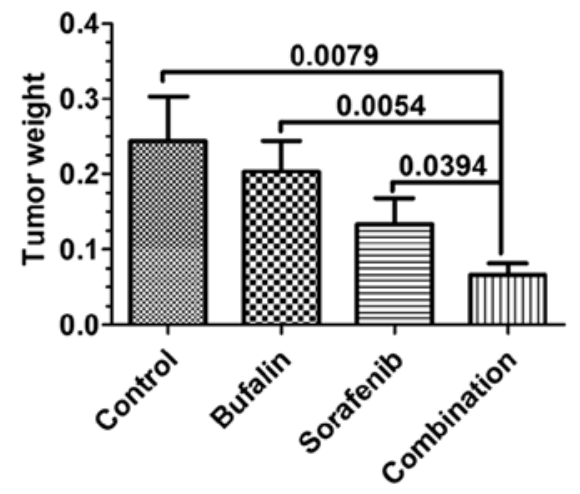

Figure 1. The combination treatment inhibits intradermal tumor angiogenesis. (A) Images of angiogenesis in intradermal tumor in nude mice with different treatments. (B) Statistical analysis of vessel numbers and branches in nude mice with different treatments. (C) Statistical analysis of the tumor weight in nude mice with different treatments.

sorafenib or bufalin and the combination at various concentrations. Sorafenib at concentrations of 2.5,5 and $10 \mu \mathrm{M}$ and bufalin at concentrations of 2.5, 5 and $10 \mathrm{nM}$ were all applied. To test their synergistic effect on the growth of HUVECs, the combination treatment with sorafenib and bufalin after $48 \mathrm{~h}$ of exposure was also performed using CCK8 assay. The combination of sorafenib and bufalin resulted in a significant inhibition in cell growth when compared with either drug alone (Fig. 2A). The combination index (CI)/fractional effect curve showed that the synergistic effects between these two agents became stronger (Fig. 2B). Besides, the morphology of HUVECs with different treatments was observed. HUVECs with no treatment were typically pebble-shaped while those with either sorafenib or bufalin stimulation resembled fibroblast morphology. In addition, the combination treatment even gendered stronger morphological changes (Fig. 2C).

Combination treatment leads to enhanced inhibition of angiogenesis in blood vessel models. The effect of the combination treatment on angiogenesis was evaluated. First, we investigated the impact of different treatments on HUVEC tubule formation. Results revealed that the combination treatment significantly decreased the formation of tubule structures compared to those from other groups (Fig. 3A). To confirm whether the combination treatment has stronger angiogenic activity in vivo, we investigated their effect in a chicken chorioallantoic membrane (CAM) model. As seen from the images, sorafenib or bufalin exerted a certain inhibitory effect in angiogenesis. However, the combination treatment successfully disrupted the vasculature and attenuated the thickness of the vessels (Fig. 3B). Next, arterial ring sprouting experiment was applied and analyzed. The result showed that under control conditions the aortic ring was able to generate neo-vessel sprouting and the density of sprouting decreased when the microvessels were incubated with either bufalin or sorafenib. The neo-vessel sprouting almost vanished when incubated with the combination (Fig. 3C).

Combination treatment leads to cell cycle arrest and apoptosis in HUVECs. As we have demonstrated the inhibitory effect of the combination treatment on angiogenesis, specific mechanisms need to be further analyzed. The percentage of cells in G2 phase increased in HUVECs treated with the combination compared to HUVECs treated with either agent alone (Fig. 4A). We then determined the effect of sorafenib, bufalin and the combination on apoptosis of HUVECs in vitro. To clarify the effect of different treatments on apoptosis in HUVECs, flow cytometry was employed. HUVECs treated with sorafenib and bufalin experienced more apoptosis than the untreated ones, as quantified by Annexin V and PI staining. Not surprisingly, the result showed that apoptotic rate was significantly higher in HUVECs treated with the combination of sorafenib and bufalin, suggesting that bufalin cooperates with sorafenib to induce apoptosis of HUVECs (Fig. 4B). A drastic increase in the apoptosis by the combination treatment was also confirmed, as determined by the quantification of 
A

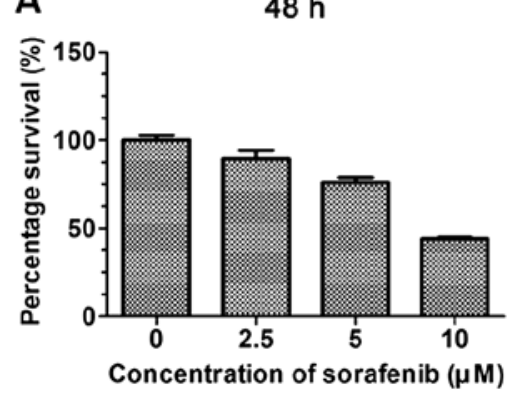

B

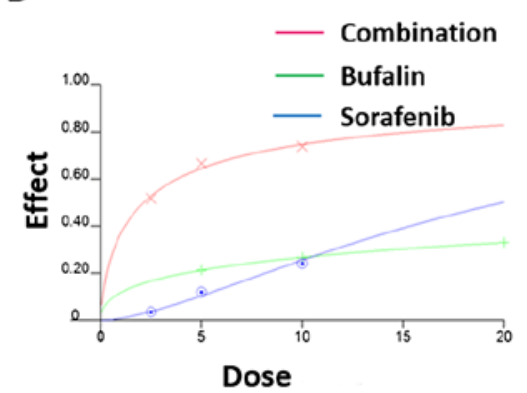

C

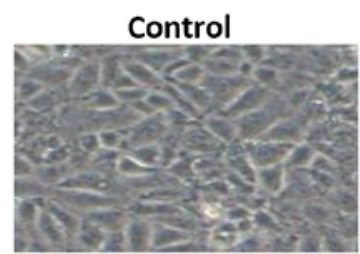

$48 \mathrm{~h}$

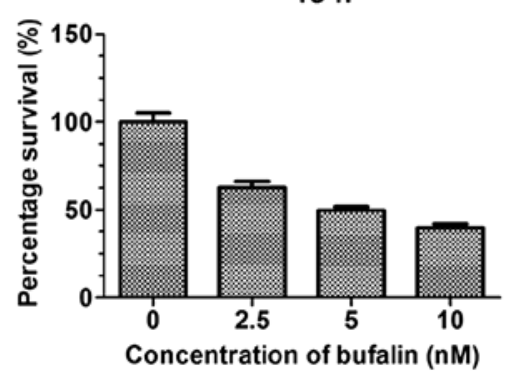

Combination-monte carlo

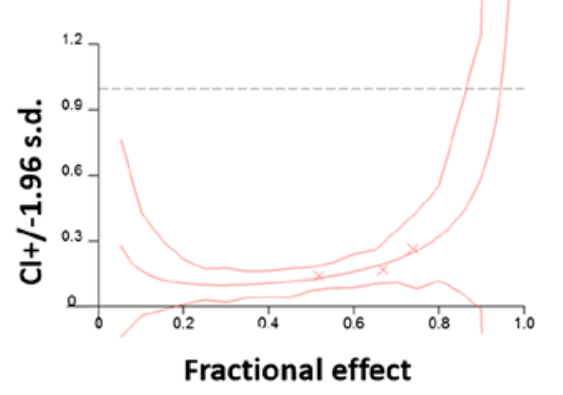

\begin{abstract}
-
\end{abstract}
$48 \mathrm{~h}$

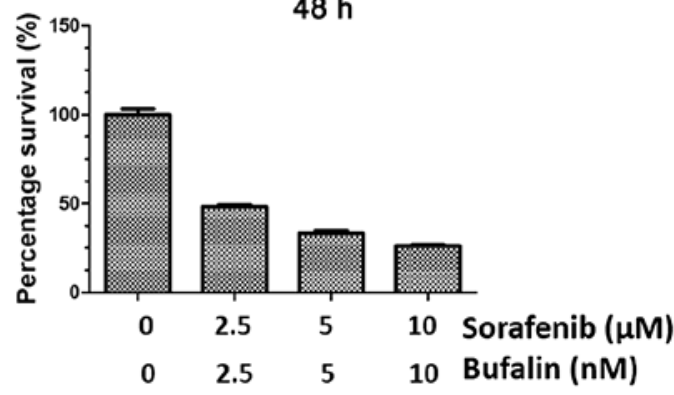

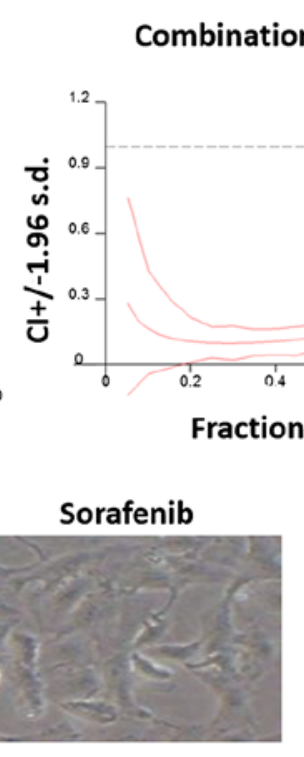

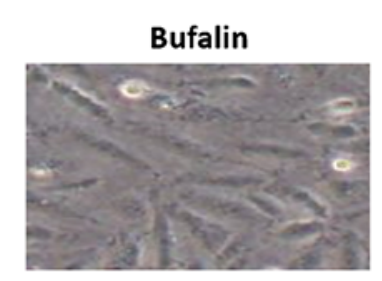

\begin{tabular}{llll}
\multicolumn{4}{c}{ Cl For HUVEC } \\
\hline $\begin{array}{l}\text { Bufalin sorafenib } \\
(\mathrm{nM})\end{array}$ & $(\mu \mathrm{M})$ & $\mathrm{Cl}$ \\
\hline 2.5 & 2.5 & 0.517 & 0.141 \\
5 & 5 & 0.668 & 0.170 \\
10 & 10 & 0.739 & 0.266 \\
\hline
\end{tabular}

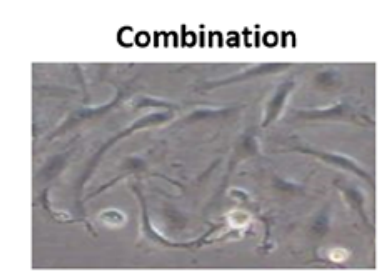

Figure 2. Synergistic inhibitory efficacy of combination treatment on proliferation of HUVEC in vitro. (A) HUVECs were treated with $2.5,5$ or $10 \mu \mathrm{M}$ sorafenib and $2.5,5$ or $10 \mathrm{nM}$ bufalin for $48 \mathrm{~h}$. HUVECs were also treated with the combination of sorafenib and bufalin at different concentrations as shown in the picture. Cell viability was determined by CCK8 assay. (B) HUVECs were treated with increasing concentrations of the indicated drugs for 48 h. The $\mathrm{CI} /$ fractional effects curve showed the synergistic anti-growth effects of the combination drugs. Multiple drug analyses were conducted by calculating CI values. (C) The morphologies of HUVECs with individual treatment of either sorafenib or bufalin were detected. The morphology of HUVECs with the combination treatment was also observed using a microscope.

fluorescent intensity of Hoechst 33258 via fluorescent microscope (Fig. 4C).

Combination treatment inhibits migration in HUVECs. Since endothelial migration plays a critical role in tumor angiogenesis, we next observed the migration ability of HUVECs with the combination treatment. Wound healing was used to study whether different treatments would impact migration of HUVECs. The combination-treated HUVECs showed decreased motility compared with the controls or single drug treatment group (Fig. 5A). Transwell assay was further used to detect the migration in HUVEC after different treatments. The combination treatment also showed a significant decrease in migration compared with the controls or single drug treatment group (Fig. 5B).

Combination-treated CM impairs HUVEC angiogenesis and the expression of angiogenesis-related cytokines in vitro. As we found that HUVECs showed increased apoptosis with the combination treatment, we assume the anti-angiogenic capacity of the combination treatment may, at least in part, be attributed to the induced apoptosis acceleration. Vessel formation is a process regulated by a network of cytokines, released from endothelial cells in an autocrine manner. These cytokines may lead to the maturation of the adjacent cells and migration, thus affecting angiogenesis. Firstly, the effect of different treatments on angiogenesis in HUVECs was investigated using the HUVEC tubule formation assay. CM derived from HUVECs with different treatments for $48 \mathrm{~h}$ were harvested. The effect of CM on tubule formation and migration of HUVECs were observed at 12, 24, 36, $48 \mathrm{~h}$ after incubation. CM derived from the combination treatment significantly decreased the formation of tubule structures compared to $\mathrm{CM}$ from other groups (Fig. 6A).

Moreover, CM from the combination treatment significantly decreased the migration capacity of HUVECs, as demonstrated in Transwell assay (Fig. 6B). Such phenomenon is a good indication of cytokine changes in HUVECs with different treatments. Thus, the conditioned media were collected and detected. Cytokines crucial for angiogenesis were detected using the Human Angiogenesis Array. The array has shown that angiogenic cytokines such as angiogenin, PDGF-BB and VEGF vary significantly between the untreated and the combination-treated group (Fig. 6C). As VEGF plays a central role in the regulation of angiogenesis, we examined the expression of VEGF in untreated and the 
A

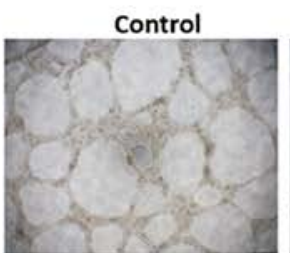

Sorafenib

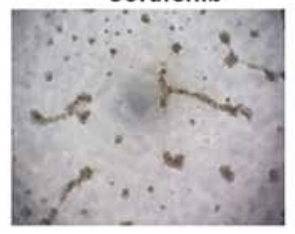

B
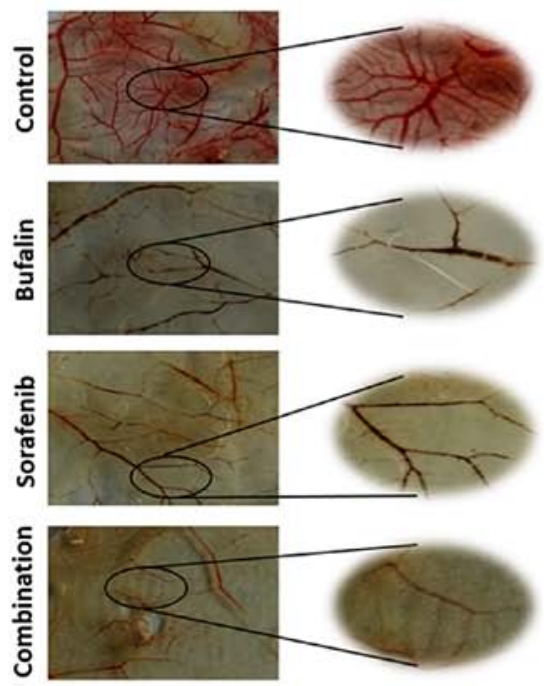

C

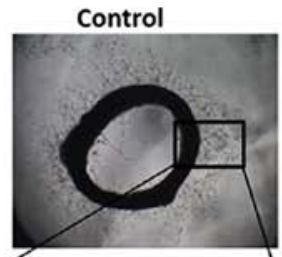

$24 \mathrm{~h}$
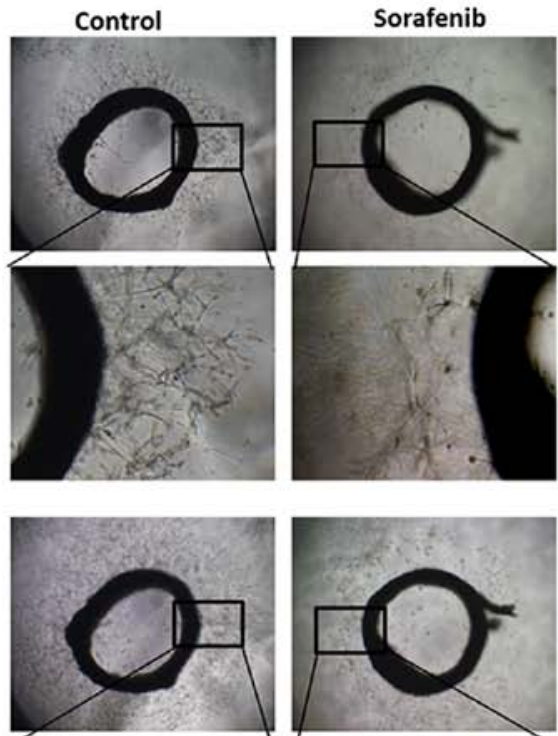

$48 \mathrm{~h}$
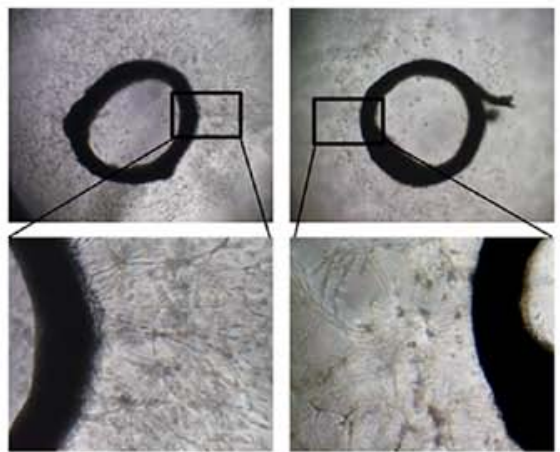
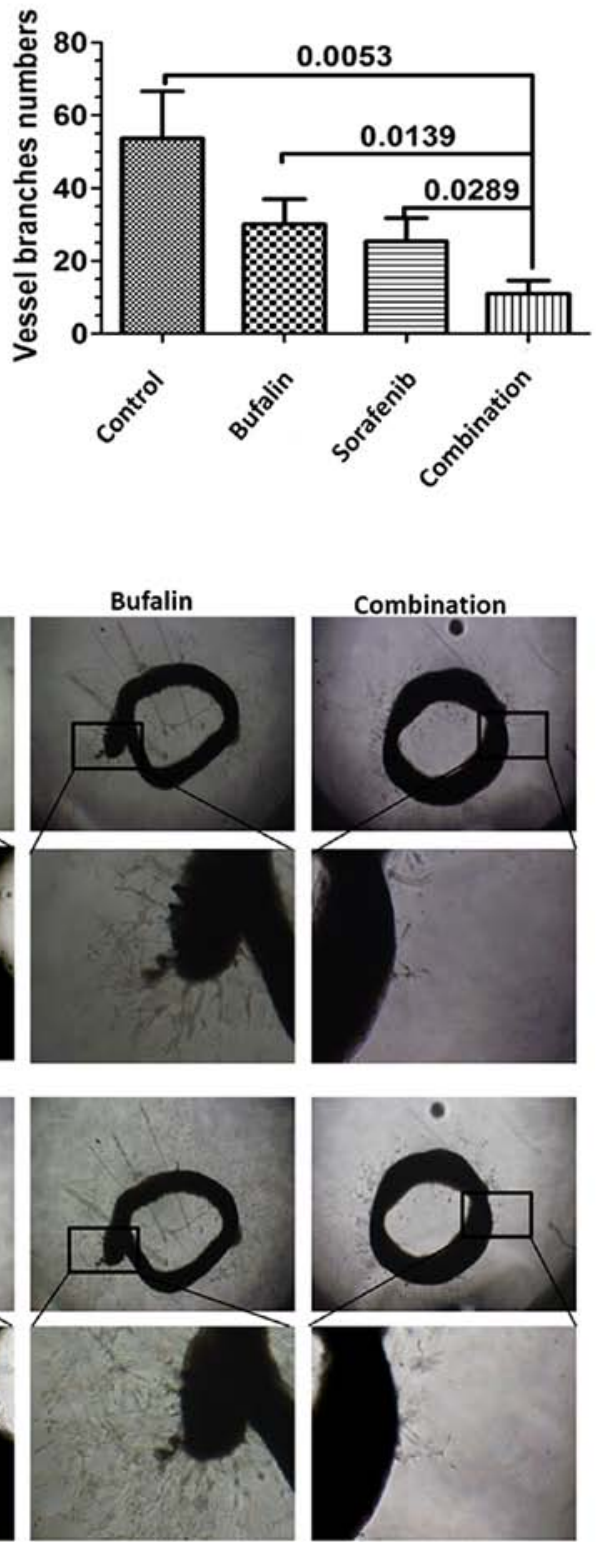

Figure 3. The combination treatment leads to enhanced inhibition of angiogenesis in blood vessel models. (A) HUVECs were subjected to different treatments. After 2 days, cells were seeded onto polymerized matrigel at $4 \times 10^{4}$ cells/well/100 $\mu 1$ in a 96 -well plate. Patterns of tube formation were photographed. (B) The anti-angiogenic effect of different treatments on CAM. The 8-day-old CAMs were treated with sorafenib, bufalin and the combination for $48 \mathrm{~h}$ and then patterns of angiogenesis were photographed. Total numbers of branches of blood vessels were analyzed. Representative results from three independent experiments, all of which gave similar results. (C) The anti-angiogenic effects of different treatments on HUVEC were observed using aortic ring assay. Aortic segments from Wistar rats were placed in Matrigel-covered wells and treated with different drugs. Photographs of sprouts from the margins of aortic rings were captured. Square frames indicate sprouting microvessels. 

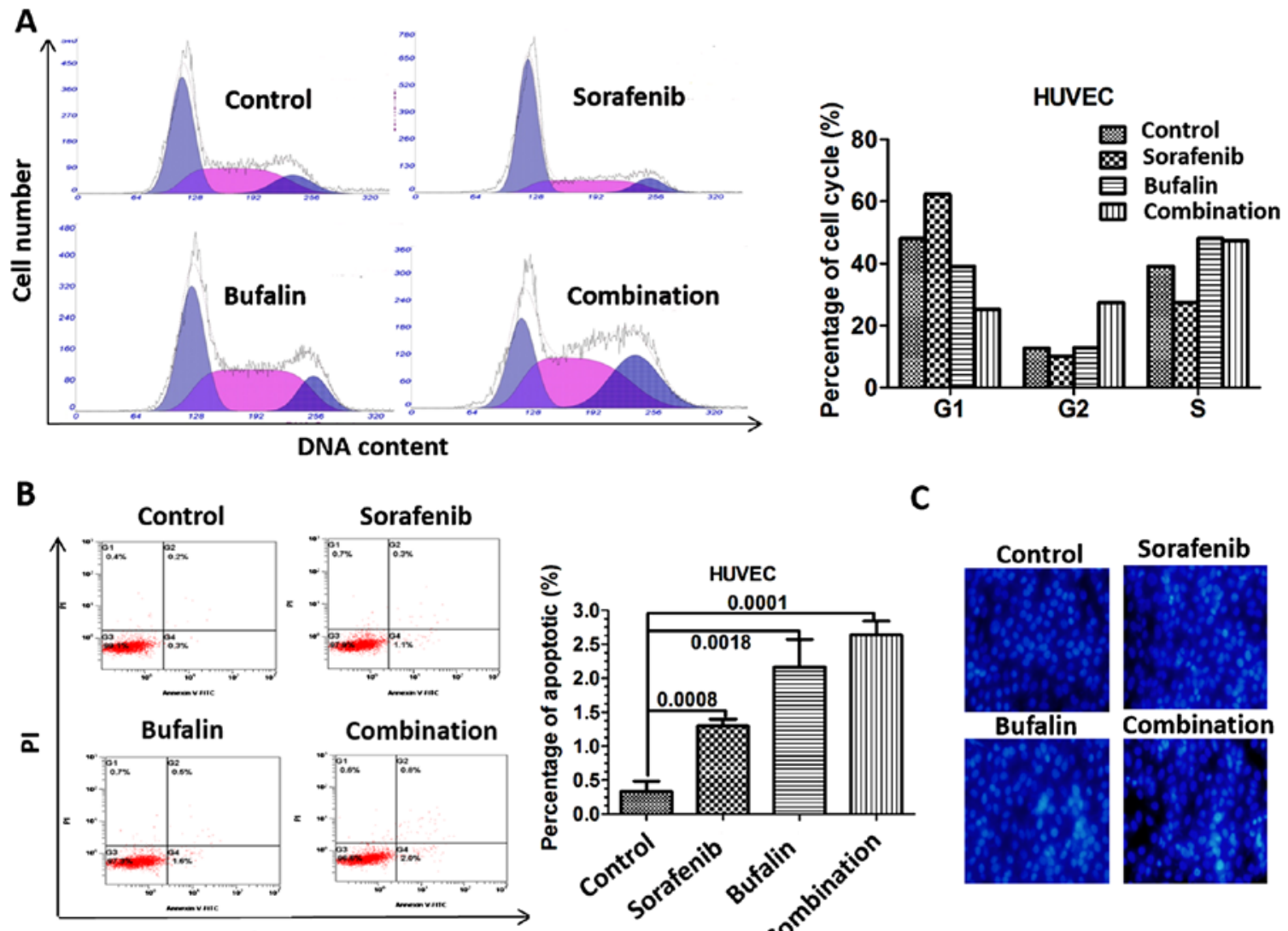

Annexin V-FITC
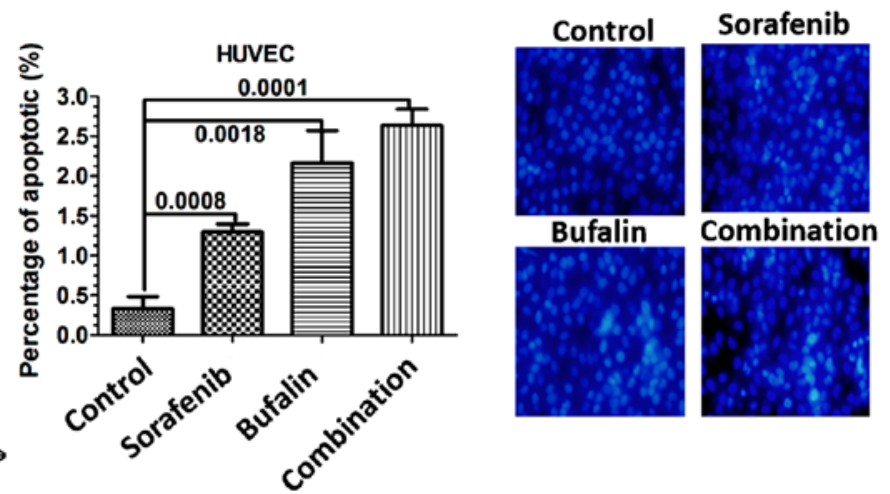

Figure 4. The combination treatment leads to cell cycle arrest and apoptosis in HUVECs. Different treatments were assessed for (A) cell cycle progression using flow cytometry, percentage of cell cycle in G1, G2, S phases were evaluated, respectively, and (B) flow cytometry was adopted to analyze the effect of different treatments on the frequency apoptosis of HUVECs by PE/7ADD staining. (C) Apoptosis was also determined by fluorescent intensity through Hoechst 33258 staining.

A
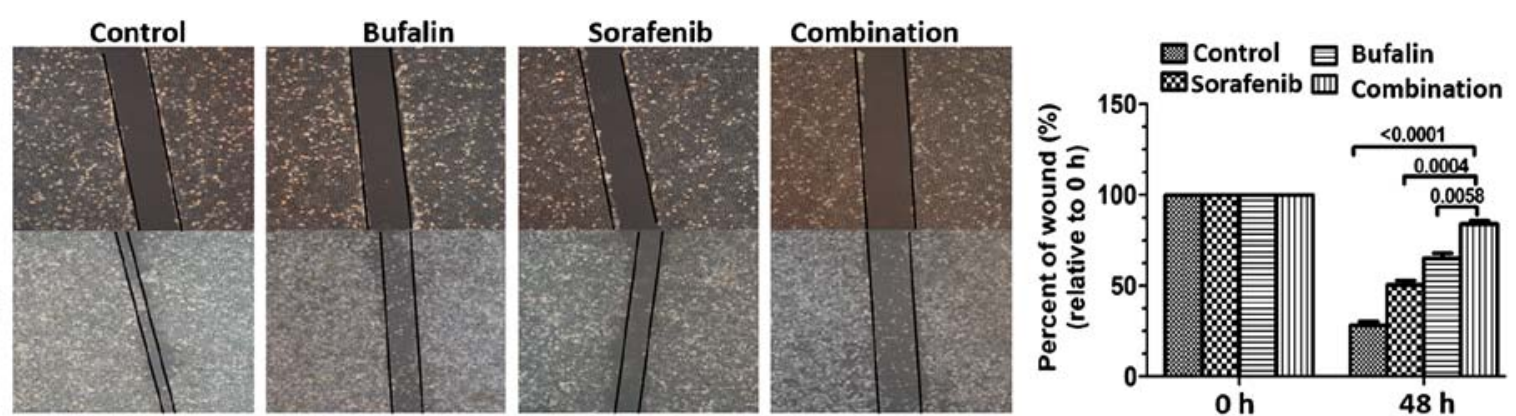

B
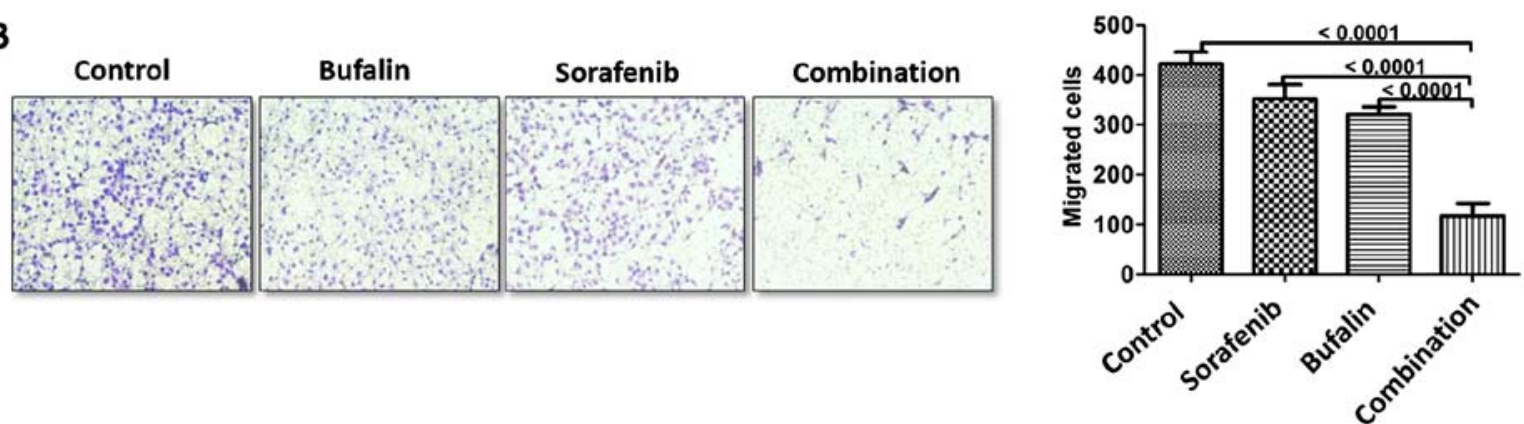

Figure 5. The combination treatment inhibits migration in HUVECs. (A) Migrations of different treatments were measured by wound healing. HUVECs were exposed to different treatments. After 2 days, cells were wounded with a pipette tip and incubated for $12 \mathrm{~h}$. The number of cells that migrated into the denuded areas were counted and are plotted as percentages relative to values for untreated control cells. Results were quantitated by counting migrated cells in five randomly chosen fields for each replicate $(n=3)$. (B) Migrations of different treatments were measured by a Transwell assay. Results were quantitated by counting migrated cells in five randomly chosen high-power fields for each replicate $(n=3)$. 

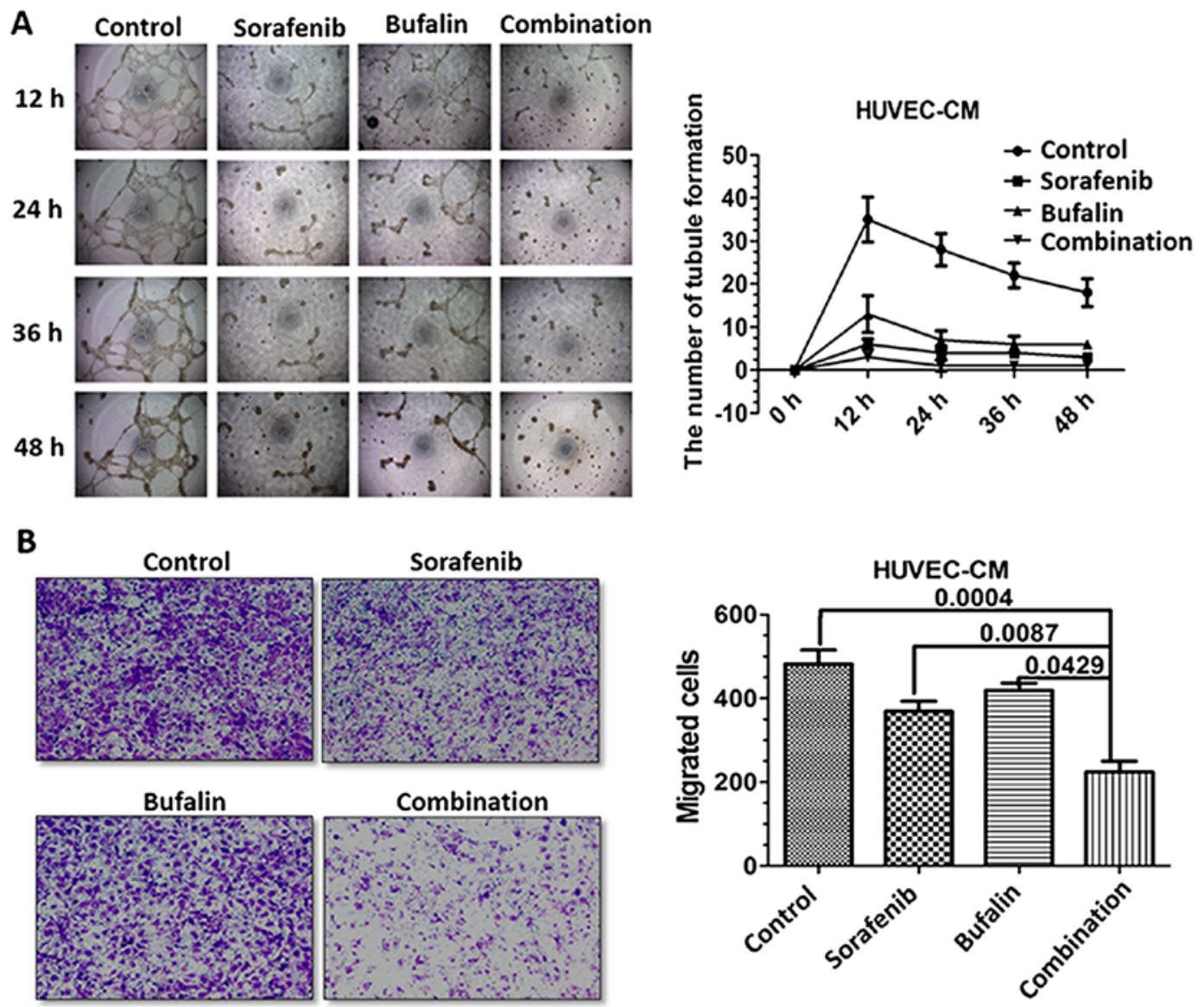

C
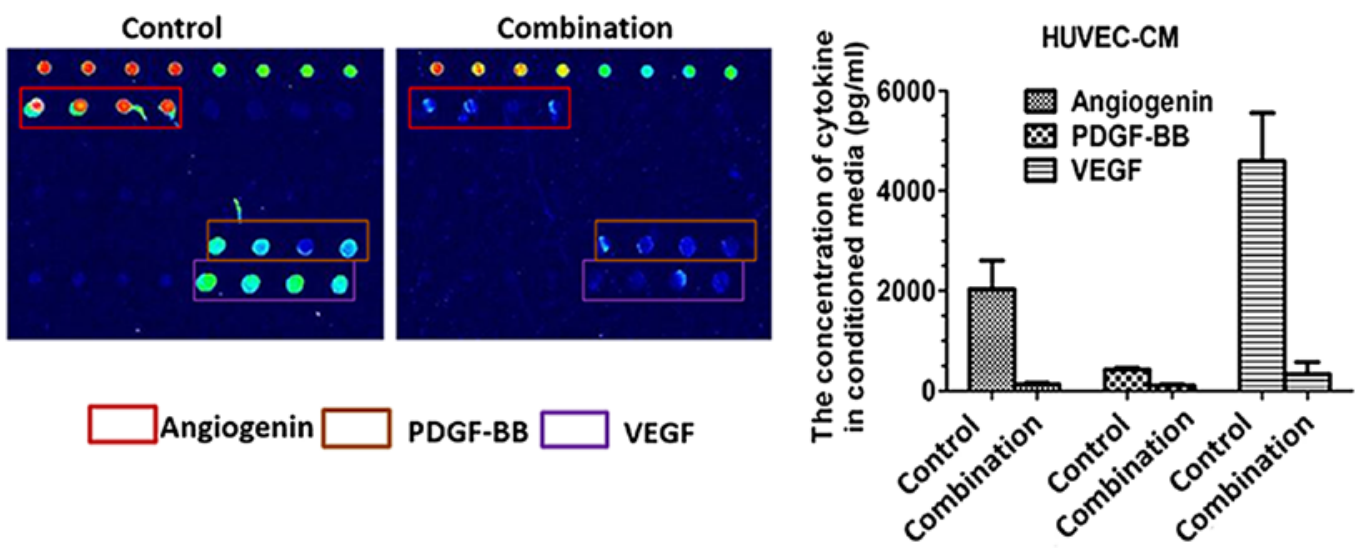

D

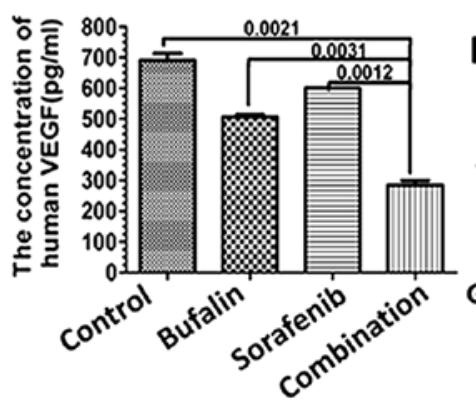

$\mathbf{E}$
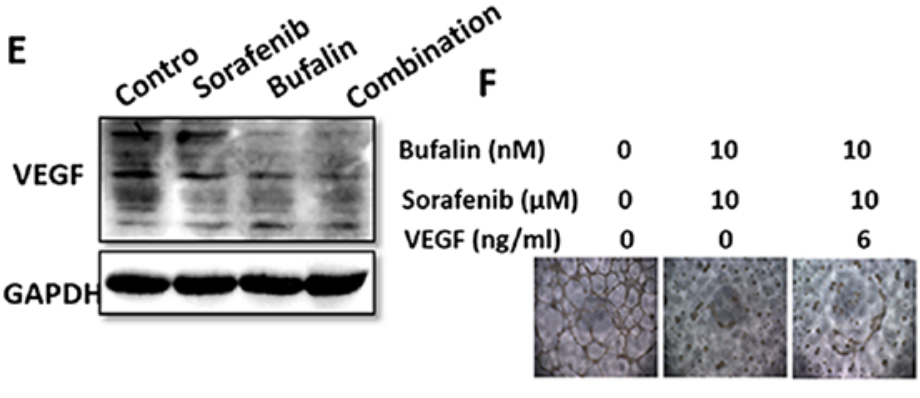

Figure 6. The combination-treated CM impairs HUVEC angiogenesis and the expression of angiogenesis-related cytokines in vitro. (A) The effects of conditioned medium extracted from different treatments on HUVECs for 12, 24, 36 and 48 h. (B) Representative images and quantification of the number of migrated HUVECs after incubation in CM derived from HUVEC with indicated treatments using the Transwell migration assay. (C) Detection of cytokines critical for angiogenesis via Human Angiogenesis Array. The concentrations of the saliently altered cytokines (Angiogenin, PDGF-BB, VEGF) were analyzed. (D) Detection of VEGF secretion in conditioned medium from HUVEC with different treatments by ELISA. (E) Western blot analysis of VEGF protein expression in HUVECs with different treatments. VEGF levels were normalized to GAPDH and expressed relative to the respective control cells. (F) VEGF antibody combined with other treatments were employed to block the secretion in HUVECs, and their effect on tube formation are shown. 
A

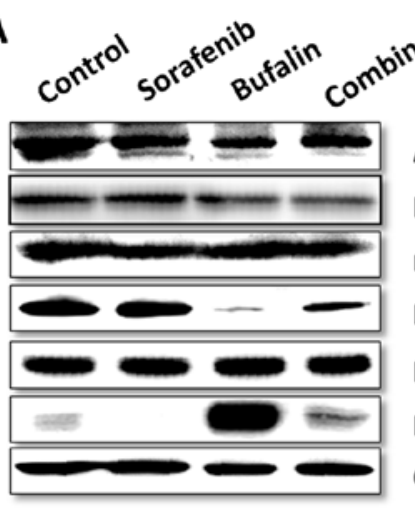

B

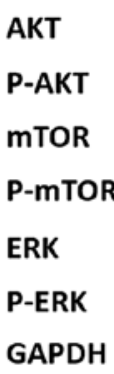

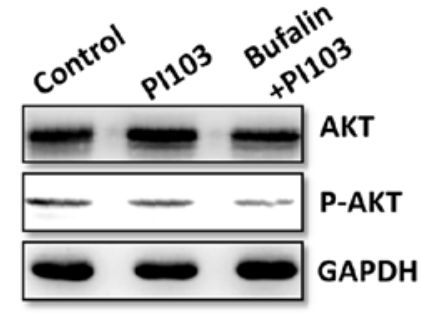

C

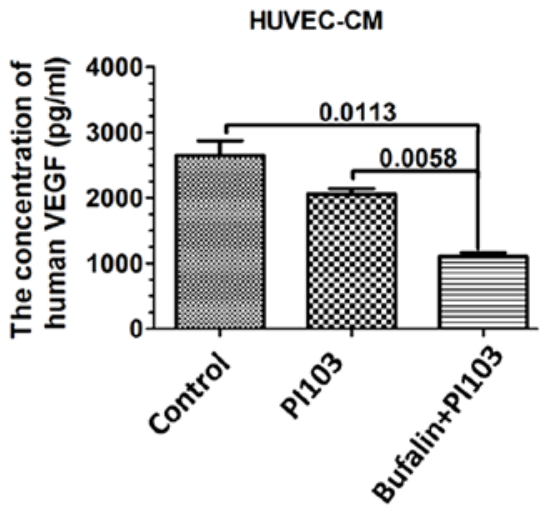

D

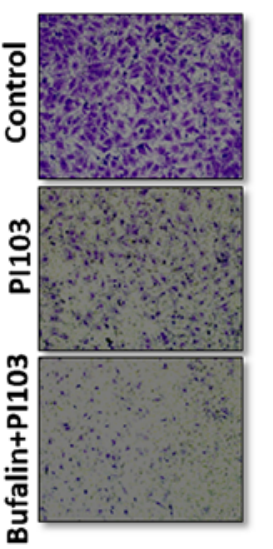

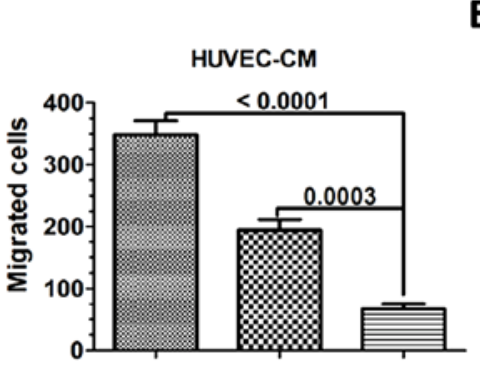

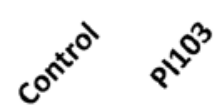

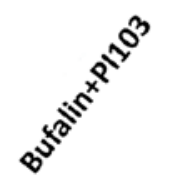

$\mathbf{E}$
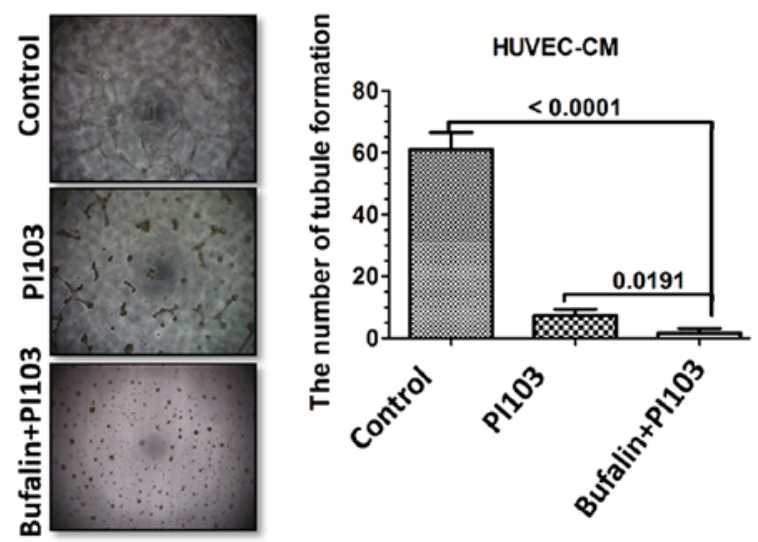

Figure 7. The combination treatment regulates VEGF-mediated HUVEC via PI3K/AKT pathway. (A) The expression of p-AKT, p-mTOR in PI3K/AKT/mTOR signaling pathway and p-ERK was detected in HUVECs with different treatments via western blotting. (B) HUVECs were treated with PI3K/AKT inhibitor, PI103, and stimulated with bufalin for $48 \mathrm{~h}$. HUVECs were harvested, and western blotting was performed for detection of p-AKT. (C) HUVECs were treated with PI3K/AKT inhibitor, PI103 $(2 \mu \mathrm{M})$, and stimulated with bufalin for $48 \mathrm{~h}$. Secretions of VEGF of different treatments were detected by ELISA. (D) HUVECs were treated with PI3K/AKT inhibitor, PI103, and stimulated with bufalin for $48 \mathrm{~h}$. Representative images of the number of migrated HUVECs after incubation in conditioned medium (CM) derived from different treatments in the Transwell migration assay are shown. (E) HUVECs were treated with PI3K/AKT inhibitor, PI103, and stimulated with bufalin for $48 \mathrm{~h}$. Representative images of the tubule formation after incubation in CM derived from different treatment and analysis of the number of tubule formation are shown.

combination-treated HUVECs. The concentration of VEGF was significantly downregulated in the medium from HUVEC with the combination treatment, as detected by ELISA (Fig. 6D). Additionally, the expression of VEGFs proteins were detected in HUVECs. The expressions of VEGFs in HUVECs as detected through western blotting were consistent with the VEGFs released from CM (Fig. 6E). Noteworthy, the addition of VEGF reversed the inhibitory effect on tube formation by the combination treatment, which was indicative of the role of VEGF in vessel formation (Fig. 6F).

Combination treatment regulates VEGF-mediated HUVEC via PI3K/AKT pathway. We have already proved that the combination treatment-induced VEGF reduction may be one of the reasons leading to attenuated angiogenesis. Therefore, the effects of different treatments on pathways regulating VEGF were analyzed. Thus, we investigated whether the AKT, mTOR and ERK pathways could be affected by the combination treatment. For this purpose, we performed western blotting to detect the activation of AKT, mTOR and ERK in HUVECs. We found that HUVECs treated with bufalin showed a significant decrease in AKT and mTOR phosphorylation and a significant increase in ERK phosphorylation compared with the control group. HUVECs treated with sorafenib showed a significant decrease in ERK phosphorylation compared with the control group. However, HUVECs treated with combination treatment showed a significant decrease in AKT phosphorylation compared with the control group or monotherapy group, implying that AKT may be involved in the collaborative medication process (Fig. 7A).

To further determine whether the AKT signaling pathway mediated VEGF expression induced by combination treatment, we treated HUVECs with the PI3K/AKT inhibitor PI103 $(2 \mu \mathrm{M})$ (Fig. 7B). We observed that VEGF expression was significantly downregulated by PI103 treatment, suggesting a role of the AKT pathway in regulating VEGF production induced by combination treatment (Fig. 7C). We next evaluated the effect of the CM treated by PI103 on HUVEC migration and tubule formation. Compared with untreated CM, HUVEC migration and tubule formation were obviously suppressed by the CM treated with PI103. Such phenomenon was even stronger in the CM treated with PI103 combined with bufalin (Fig. 7D and E).

Combination treatment inhibits tumor growth and tumor angiogenesis in vivo. To further investigate whether bufalin 


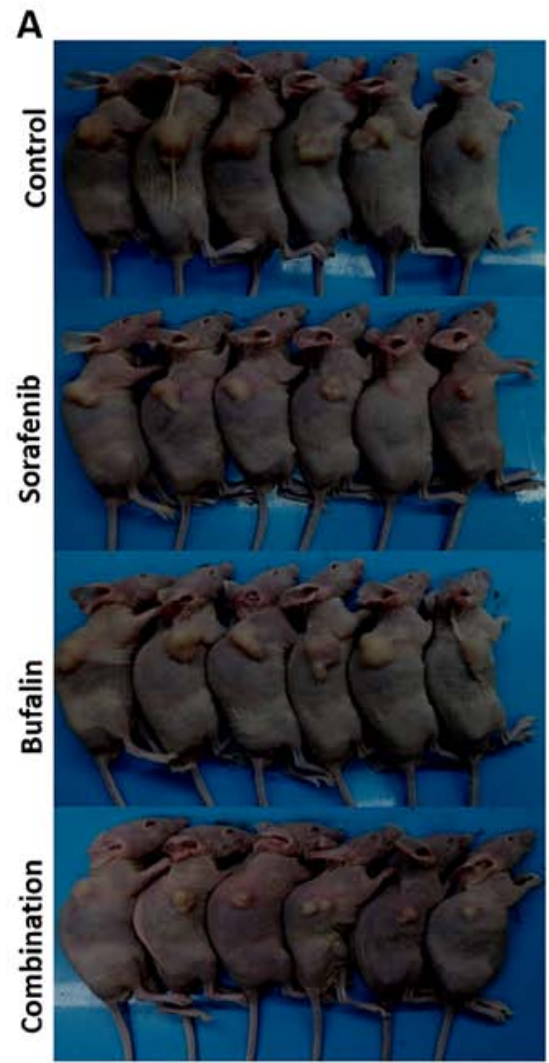

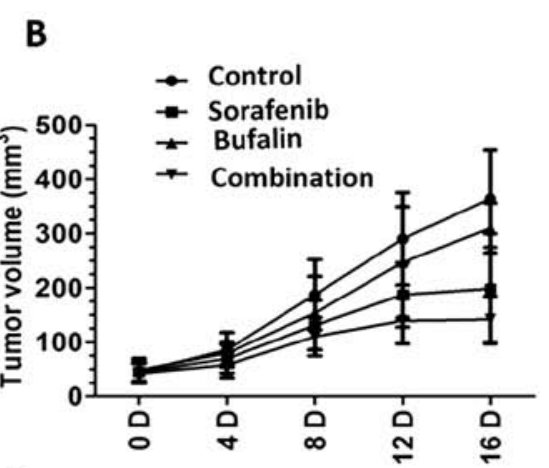

D
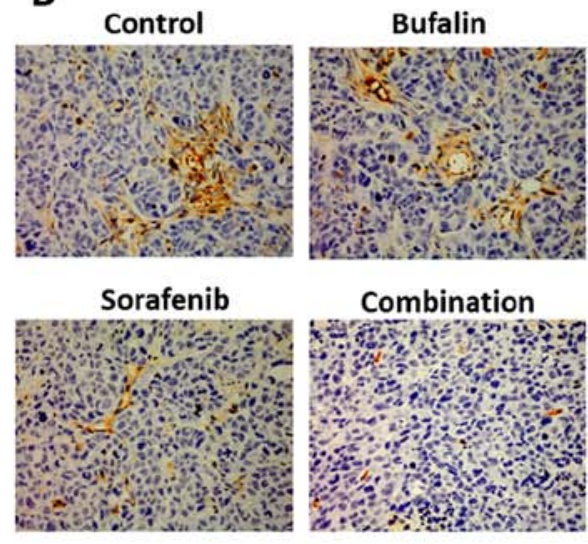

C
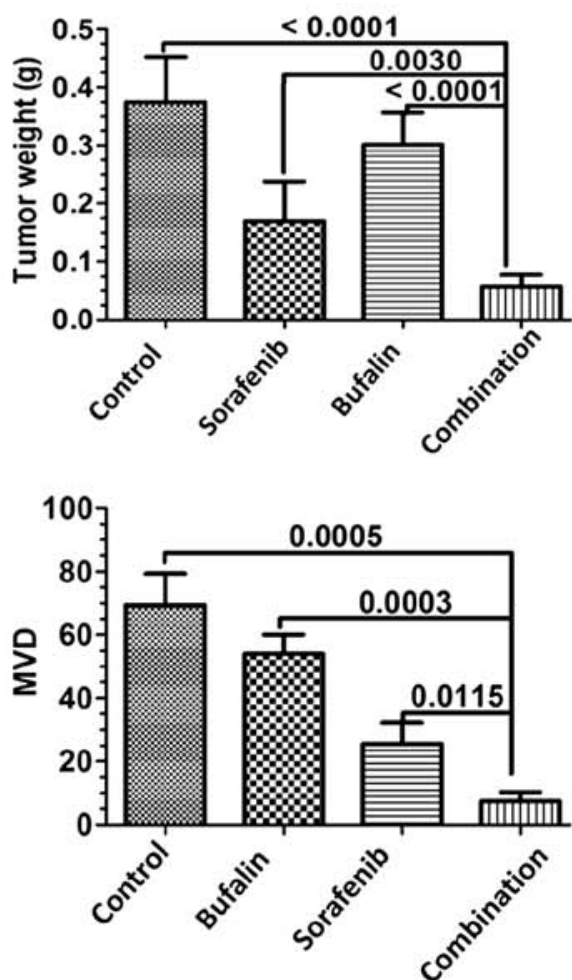

Figure 8. The combination treatment inhibits tumor growth and tumor angiogenesis in vivo. Mice with SMMC7721 human xenografts were randomly divided into four groups and given indicated treatments five days a week for a period of two weeks. The tumors were then removed for analysis. (A) Tumors from the mice treated with the combination drugs were smaller than those from other groups. (B) Tumor volumes were significantly smaller in the combination group than in any other group. (C) Tumor weights were significantly lower in the combination group than in any other group. (D) Immunohistochemical staining of CD31 was used to quantify the microvessel density (MVD).

facilitates the sorafenib antitumor activity in vivo, HCC tumor xenografts were established in BALBc nu/nu mice. As shown in Fig. 8A, sorafenib combined with bufalin inhibited the growth of HCC-derived tumors more potently than the individual group (Fig. 8A). Tumor volumes were smaller in the combination-treated group than the individual group (Fig. 8B). Tumor weights of mice of all the groups were analyzed. As shown in Fig. 8C, mice with the combination treatment showed lower weights of tumor, as compared to the other groups (Fig. 8C). Since CD31 is the prominent endothelial marker which binds specifically to blood microvessels, the tumors were then evaluated for expression of CD31 by immunohistochemical analysis. The combination treatment group showed reduced microvessel density (MVD) more than any other group (Fig. 8D).

\section{Discussion}

At present, $\mathrm{HCC}$ remains a considerable challenge in clinical practice, and current treatments are inadequate (29). Abundant blood supply has been noted in HCC, which leads to the application of many anti-angiogenic drugs in HCC treatments.

Sorafenib, a commonly known anti-angiogenic tyrosine kinase inhibitor, has been approved for liver cancer. Approved by the FDA to treat unresectable HCC, sorafenib is currently the main drug used in the treatment of patients with advanced HCC (23). It is a small molecule that inhibits tumor angio- genesis. It acts by inhibiting the serine-threonine kinases Raf-1 and B-Raf and the receptor tyrosine kinase activity of vascular endothelial growth factor receptors (VEGFRs) 1,2 and 3 and platelet-derived growth factor receptor $\beta$. Sorafenib targets VEGF receptors, and is now thought to exert its effect primarily by blocking VEGF signaling, as its efficacy against B-Raf is questionable. Although sorafenib has been shown to improve overall survival from a median of 7.9 to 10.7 months in patients with advanced HCC, its effect remains to be improved (24). Therefore, recently attention has been focused on the finding and development of potent angiogenesis inhibitors.

Bufalin, one of the prominent components of bufadienolides, was reported to treat various tumors by inducing apoptosis, inhibiting proliferation and matastasis (25). However, there are few investigations concerning the effect of bufalin on angiogenesis. An earlier study demonstrated that the in vitro angioinhibitory action of bufalin may be induced by the proliferation inhibition of endothelial cells through the arrest at the G2/M phase of a cell cycle (26). Thus, we hypothesized that they may exert more potent efficacy against angiogenesis than either alone.

In the present study, we first examined the effect of bufalin and sorafenib on angiogenesis of human HCC intradermal tumor in nude mice. Results confirmed that the combination treatment significantly inhibited tumor angiogenesis compared with mice administered the vehicle or the individual treat- 
ment. Next, we demonstrated that the combination application significantly suppressed vessel formation as demonstrated in the tube formation, chick chorioallantoic membrane and rat aortic rings. As tumor angiogenesis can be modulated through apoptosis and migration of endothelial cells, we anticipated that apoptosis and migration alteration could be discovered in HUVECs with the combination treatment. The assumption was validated, as tested in the apoptosis and migration assay.

Considering the pro-angiogenic role of cytokines released from endothelial cells, suspensions from HUVECs with different treatments were collected as CM. The combinationtreated CM significantly inhibited the migration of HUVEC cells and blood vessels formation in vitro. Of note, multiple cytokines associated with angiogenesis were downregulated in the combination-treated CM, as detected by Human Angiogenesis Array, among which VEGF was the most saliently downregulated. The inhibition of PI3K/AKT pathway upon the combination treatment was observed, as evidenced by the evaluation of p-AKT. Finally, we revealed that bufalininduced VEGF reduction may be attributed to the inhibition of the PI3K/AKT pathway, using PI3K inhibitor. Together, our study may provide better insight of the application of sorafenib in combination with bufalin.

Over the years, researchers have developed a wide range of experimental and integrative approaches, including conventional migration and proliferation assays, tubule formation assays, the chick chorioallantoic membrane (CAM) assay, the aortic ring and many other methods, to investigate the process of angiogenesis (30). Among them, the model of intradermal tumor angiogenesis was adopted according to previous methods, as it may provide a more direct and vivid result (28). Our results showed that the combination treatment achieved enhanced effect against tumor angiogenesis, as distinctly observed in this model.

It has been demonstrated that VEGF secreted by stromal cells such as endothelial cells has multiple functions (31). VEGF functions as a primary stimulus for angiogenesis, which is a process that involves the ability of VEGF receptors to stimulate signaling pathways that induce the proliferation and the migration of endothelial cells (32). The prevailing idea is that therapies target angiogenesis and endothelial cell functions, and this aspect of VEGFtargeted therapy has been extensively studied $(14,33,34)$. VEGF binds to VEGF receptor, which leads to the activation of phosphatidylinositol 3-kinase (PI3K)/AKT signaling pathway. PI3K/AKT signaling regulates angiogenesis through affecting the expression of VEGF $(9-12,14)$. In addition, forced expression of PI3K alone is sufficient to increase angiogenesis via increased VEGF expression. The phospholipid second messengers generated by PI3K provide a common mechanism for multiple steps during angiogenesis $(35,36)$. Serine-threonine protein kinase AKT is a major downstream target of PI3K for regulating tumor growth and angiogenesis $(37,38)$. For instance, $\mathrm{PI} 3 \mathrm{~K} / \mathrm{AKT}$ may regulate angiogenesis by several downstream targets such as mTOR/p70S6K1, FOXO, NOS, and GSK-3 $\beta$ (39-41).A study has reported that Arnebin-1 promotes angiogenesis by inducing eNOS, VEGF and HIF-1 expression through the PI3K-dependent pathway (42).
In this study, we found that sorafenib was able to slightly decrease the level of VEGF expression in HUVECs. As expected, such phenomenon was more salient when combined with bufalin, as evidenced by VEGF expression in the HUVEC and HUVEC-CM with different treatments. However, the exact mechanism of the bufalin regulation of VEGF expression remains to be elucidated. Previous studies have reported that PI3K/AKT stimulation of angiogenesis is mediated, in part, by mTOR and HIF-1 (43-45). Sustained endothelial activation of AKT has been shown to induce the formation of structurally and functionally abnormal blood vessels that recapitulate the aberrations of tumor vessels (46). Besides, inhibition of AKT signaling was able to inhibit the vascularization (47).

In search of mechanisms underlying the process, we tested the effect of different treatments on p-AKT and p-mTOR in $\mathrm{PI} 3 \mathrm{~K} / \mathrm{AKT} / \mathrm{mTOR}$ pathway and $\mathrm{p}-\mathrm{ERK}$ expression. We found that $\mathrm{p}-\mathrm{AKT}$ was decreased while p-ERK was increased by the combination treatment. It has been reported that p-AKT and ERK enhance the expression of pro-angiogenesis target genes, such as VEGF, and then promote endothelial cell migration and proliferation, thus contributing to tumor angiogenesis (48-50). The seemingly paradoxical phenomenon that the combination treatment enhances ERK phosphorylation can be explained by the following: i) The combination-evoked increase in p-ERK may be due to ER stress. ii) Its inhibition on other pathways contributing to angiogenesis is more potent than its effect on p-ERK activation, thus may offset this proangiogenesis process. iii) Although the phosporylation of ERK may upregulate VEGF expression, it may downregulate other angiogenic factors potently, thus resulting in inhibition of angiogenesis.

The PI3K inhibition with PI103 was used to determine whether the PI3K pathway has a role in AKT and VEGF expression and whether combination treatment can decrease VEGF expression via inhibiting the PI3K/AKT pathway. VEGF expression was almost completely abolished by PI103, which suggest that the PI3K pathway is a positive regulator of VEGF expression. The suppression of HUVEC migration and tube formation by PI103, the PI3K inhibitor, as well as bufalin implies that combination treatment may inhibit VEGF expression via the PI3K/AKT pathway.

Collectively, the present study aids the understanding of the antitumor effect of bufalin in HCC in terms of its anti-angiogenic effect on HUVECs, which may support a breakthrough in the use of TCM for the treatment of HCC. It also uncovered an important functional role of bufalin in enhancing the anti-angiogenic effect of sorafenib, suggesting that bufalin combined with sorafenib can serve as an effective treatment for anti-angiogenesis therapy in HCC.

\section{Acknowledgements}

We thank Huiying Chi (Shanghai Geriatric Institute of Chinese Medicine, Longhua Hospital, Shanghai University of Traditional Chinese Medicine, Shanghai) for the technical help in the experiments. This study was supported jointly by the National Natural Science Foundation of China (no. 81273954) and the Hundred Talents Program of the Health System in Shanghai (no. JGRC1302). 


\section{References}

1. Sun HC and Tang ZY: Angiogenesis in hepatocellular carcinoma: The retrospectives and perspectives. J Cancer Res Clin Oncol 130: 307-319, 2004

2. Folkman J: Angiogenesis: An organizing principle for drug discovery? Nat Rev Drug Discov 6: 273-286, 2007.

3. Van de Veire S, Stalmans I, Heindryckx F, Oura H, TijerasRaballand A, Schmidt T, Loges S, Albrecht I, Jonckx B, Vinckier S, et al: Further pharmacological and genetic evidence for the efficacy of PlGF inhibition in cancer and eye disease. Cell 141: 178-190, 2010.

4. Weis SM and Cheresh DA: Tumor angiogenesis: Molecular pathways and therapeutic targets. Nat Med 17: 1359-1370, 2011.

5. Sharma PS, Sharma R and Tyagi T: VEGF/VEGFR pathway inhibitors as anti-angiogenic agents: Present and future. Curr Cancer Drug Targets 11: 624-653, 2011.

6. Carmeliet P and Jain RK: Angiogenesis in cancer and other diseases. Nature 407: 249-257, 2000.

7. Ribatti D, Ranieri G, Basile A, Azzariti A, Paradiso A and Vacca A: Tumor endothelial markers as a target in cancer. Expert Opin Ther Targets 16: 1215-1225, 2012.

8. Avramis IA, Kwock R and Avramis VI: Taxotere and vincristine inhibit the secretion of the angiogenesis inducing vascular endothelial growth factor (VEGF) by wild-type and drug-resistant human leukemia T-cell lines. Anticancer Res 21: 2281-2286, 2001

9. Lee JH, Choi S, Lee Y, Lee HJ, Kim KH, Ahn KS, Bae H, Lee HJ, Lee EO, Ahn KS, et al: Herbal compound farnesiferol C exerts antiangiogenic and antitumor activity and targets multiple aspects of VEGFR1 (Flt1) or VEGFR2 (Flk1) signaling cascades. Mol Cancer Ther 9: 389-399, 2010.

10. Yue GG, Fan JT, Lee JK, Zeng GZ, Ho TW, Fung KP, Leung PC Tan NH and Lau CB: Cyclopeptide RA-V inhibits angiogenesis by down-regulating ERK1/2 phosphorylation in HUVEC and HMEC-1 endothelial cells. Br J Pharmacol 164: 1883-1898, 2011.

11. Li R, Zhao $\mathrm{H}$ and Lin $\mathrm{Y}$ : Anti-tumor effect and protective effect on chemotherapeutic damage of water soluble extracts from Hedyotis diffusa. J Chin Pharmaceut Sci 11: 54-58, 2002. http://118.145.16.238/Jwk_zgyxen/EN/abstract/abstract509.shtml

12. Gupta S, Zhang D, Yi J and Shao J: Anticancer activities of Oldenlandia diffusa. J Herb Pharmacother 4: 21-33, 2004.

13. Italiano JE Jr, Richardson JL, Patel-Hett S, Battinelli E, Zaslavsky A, Short S, Ryeom S, Folkman J and Klement GL: Angiogenesis is regulated by a novel mechanism: Pro- and antiangiogenic proteins are organized into separate platelet alpha granules and differentially released. Blood 111: 1227-1233, 2008.

14. Kerbel RS: Tumor angiogenesis. N Engl J Med 358: 2039-2049, 2008.

15. Shiojima I and Walsh K: Role of Akt signaling in vascular homeostasis and angiogenesis. Circ Res 90: 1243-1250, 2002.

16. Kandel ES and Hay N: The regulation and activities of the multifunctional serine/threonine kinase Akt/PKB. Exp Cell Res 253 210-229, 1999.

17. Mazure NM, Chen EY, Laderoute KR and Giaccia AJ: Induction of vascular endothelial growth factor by hypoxia is modulated by a phosphatidylinositol 3-kinase/Akt signaling pathway in Ha-ras-transformed cells through a hypoxia inducible factor-1 transcriptional element. Blood 90: 3322-3331, 1997.

18. Arsham AM, Plas DR, Thompson CB and Simon MC: Akt and hypoxia-inducible factor-1 independently enhance tumor growth and angiogenesis. Cancer Res 64: 3500-3507, 2004.

19. Sun JF, Phung T, Shiojima I, Felske T, Upalakalin JN, Feng D, Kornaga T, Dor T, Dvorak AM, Walsh K, et al: Microvascular patterning is controlled by fine-tuning the Akt signal. Proc Natl Acad Sci USA 102: 128-133, 2005.

20. Shiojima I, Sato K, Izumiya Y, Schiekofer S, Ito M, Liao R, Colucci WS and Walsh K: Disruption of coordinated cardiac hypertrophy and angiogenesis contributes to the transition to heart failure. J Clin Invest 115: 2108-2118, 2005.

21. Nagoshi T, Matsui T, Aoyama T, Leri A, Anversa P, Li L, Ogawa W, del Monte F, Gwathmey JK, Grazette L, et al: PI3K rescues the detrimental effects of chronic Akt activation in the heart during ischemia/reperfusion injury. J Clin Invest 115 : 2128-2138, 2005.

22. Zhang Q, Oh CK, Messadi DV, Duong HS, Kelly AP, Soo C, Wang L and Le AD: Hypoxia-induced HIF-1 alpha accumulation is augmented in a co-culture of keloid fibroblasts and human mast cells: Involvement of ERK1/2 and PI-3K/Akt. Exp Cell Res 312: $145-155,2006$.
23. Llovet JM, Ricci S, Mazzaferro V, Hilgard P, Gane E, Blanc JF, de Oliveira AC, Santoro A, Raoul JL, Forner A, et al; SHARP Investigators Study Group: Sorafenib in advanced hepatocellular carcinoma. N Engl J Med 359: 378-390, 2008.

24. Wilhelm S, Carter C, Lynch M, Lowinger T, Dumas J, Smith RA, Schwartz B, Simantov R and Kelley S: Discovery and development of sorafenib: A multikinase inhibitor for treating cancer. Nat Rev Drug Discov 5: 835-844, 2006.

25. Meng Z, Yang P, Shen Y, Bei W, Zhang Y, Ge Y, Newman RA, Cohen L, Liu L, Thornton B, et al: Pilot study of huachansu in patients with hepatocellular carcinoma, non-small-cell lung cancer, or pancreatic cancer. Cancer 115: 5309-5318, 2009.

26. Lee DY, Yasuda M, Yamamoto T, Yoshida T and Kuroiwa Y: Bufalin inhibits endothelial cell proliferation and angiogenesis in vitro. Life Sci 60: 127-134, 1997.

27. Gao Y, Li HX, Xu LT, Wang P, Xu LY, Cohen L, Yang PY, Gu K and Meng ZQ: Bufalin enhances the anti-proliferative effect of sorafenib on human hepatocellular carcinoma cells through downregulation of ERK. Mol Biol Rep 39: 1683-1689, 2012.

28. Wedge SR, Ogilvie DJ, Dukes M, Kendrew J, Chester R, Jackson JA, Boffey SJ, Valentine PJ, Curwen JO, Musgrove HL, et al: ZD6474 inhibits vascular endothelial growth factor signaling, angiogenesis, and tumor growth following oral administration. Cancer Res 62: 4645-4655, 2002.

29. Ferlay J, Shin HR, Bray F, Forman D, Mathers C and Parkin DM: Estimates of worldwide burden of cancer in 2008: GLOBOCAN 2008. Int J Cancer 127: 2893-2917, 2010.

30. Auerbach R, Lewis R, Shinners B, Kubai L and Akhtar N: Angiogenesis assays: A critical overview. Clin Chem 49: 32-40, 2003.

31. Koch S, Tugues S, Li X, Gualandi L and Claesson-Welsh L: Signal transduction by vascular endothelial growth factor receptors. Biochem J 437: 169-183, 2011.

32. Ferrara N: VEGF as a therapeutic target in cancer. Oncology 69 (Suppl 3): 11-16, 2005.

33. Carmeliet P and Jain RK: Molecular mechanisms and clinical applications of angiogenesis. Nature 473: 298-307, 2011.

34. Olsson AK, Dimberg A, Kreuger J and Claesson-Welsh L: VEGF receptor signalling - in control of vascular function. Nat Rev Mol Cell Biol 7: 359-371, 2006.

35. Skinner HD, Zheng JZ, Fang J, Agani F and Jiang BH: Vascular endothelial growth factor transcriptional activation is mediated by hypoxia-inducible factor 1alpha, HDM2, and p70S6K1 in response to phosphatidylinositol 3-kinase/AKT signaling. J Biol Chem 279: 45643-45651, 2004.

36. Graupera M, Guillermet-Guibert J, Foukas LC, Phng LK, Cain RJ, Salpekar A, Pearce W, Meek S, Millan J, Cutillas PR, et al: Angiogenesis selectively requires the p110alpha isoform of PI3K to control endothelial cell migration. Nature 453: 662-666, 2008.

37. Staal SP: Molecular cloning of the akt oncogene and its human homologues AKT1 and AKT2: Amplification of AKT1 in a primary human gastric adenocarcinoma. Proc Natl Acad Sci USA 84: 5034-5037, 1987.

38. Tanaka H, Fujita $\mathrm{N}$ and Tsuruo T: 3-Phosphoinositide-dependent protein kinase-1-mediated IkappaB kinase beta (IkkB) phosphorylation activates NF-kappaB signaling. J Biol Chem 280: 40965-40973, 2005.

39. Gómez-Raposo C, Mendiola M, Barriuso J, Casado E, Hardisson D and Redondo A: Angiogenesis and ovarian cancer. Clin Transl Oncol 11: 564-571, 2009.

40. Ellis L, Hammers H and Pili R: Targeting tumor angiogenesis with histone deacetylase inhibitors. Cancer Lett 280: 145-153, 2009.

41. Li Q, Michaud M, Canosa S, Kuo A and Madri JA: GSK-3ß: A signaling pathway node modulating neural stem cell and endothelial cell interactions. Angiogenesis 14: 173-185, 2011.

42. Zeng Z, Huang WD, Gao Q, Su ML, Yang YF, Liu ZC and Zhu BH: Arnebin-1 promotes angiogenesis by inducing eNOS, VEGF and HIF-1 $\alpha$ expression through the PI3K-dependent pathway. Int J Mol Med 36: 685-697, 2015.

43. Marimpietri D, Nico B, Vacca A, Mangieri D, Catarsi P, Ponzoni M and Ribatti D: Synergistic inhibition of human neuroblastoma-related angiogenesis by vinblastine and rapamycin. Oncogene 24: 6785-6795, 2005.

44. Nakamura K, Martin KC, Jackson JK, Beppu K, Woo CW and Thiele CJ: Brain-derived neurotrophic factor activation of TrkB induces vascular endothelial growth factor expression via hypoxia-inducible factor-1alpha in neuroblastoma cells. Cancer Res 66: 4249-4255, 2006. 
45. Jiang BH, Jiang G, Zheng JZ, Lu Z, Hunter T and Vogt PK Phosphatidylinositol 3-kinase signaling controls levels of hypoxia-inducible factor 1. Cell Growth Differ 12: 363-369, 2001.

46. Bajaj A, Zheng Q, Adam A, Vincent P and Pumiglia K: Activation of endothelial ras signaling bypasses senescence and causes abnormal vascular morphogenesis. Cancer Res 70: 3803-3812, 2010.

47. Phung TL, Ziv K, Dabydeen D, Eyiah-Mensah G, Riveros M, Perruzzi C, Sun J, Monahan-Earley RA, Shiojima I, Nagy JA, et al: Pathological angiogenesis is induced by sustained Akt signaling and inhibited by rapamycin. Cancer Cell 10: 159-170, 2006.
48. Takahashi T, Yamaguchi S, Chida K and Shibuya M: A single autophosphorylation site on KDR/Flk-1 is essential for VEGF-Adependent activation of PLC-gamma and DNA synthesis in vascular endothelial cells. EMBO J 20: 2768-2778, 2001.

49. Jiang BH and Liu LZ: PI3K/PTEN signaling in angiogenesis and tumorigenesis. Adv Cancer Res 102: 19-65, 2009.

50. Claesson-Welsh L and Welsh M: VEGFA and tumour angiogenesis. J Intern Med 273: 114-127, 2013. 